\title{
MATRIX APPLICATIONS OF A QUADRATIC IDENTITY FOR DECOMPOSABLE SYMMETRIZED TENSORS ${ }^{1}$
}

BY MARVIN MARCUS

Communicated by A. S. Householder, November 18, 1964

1. Preliminaries. The Plücker quadratic relations $[3$, p. 312], [1, p. 52] are examples of necessary and sufficient conditions for a set of

$$
\left(\begin{array}{l}
n \\
m
\end{array}\right)
$$

numbers to be the coefficients in the expansion of a decomposable skew-symmetric tensor on an orthonormal basis of decomposable skew-symmetric tensors in the symmetry class $\Lambda^{m} V$.

The purposes of this announcement are to state necessary relations that obtain among coordinates of decomposable tensors in an arbitrary symmetry class of tensors (Theorem 1) and to indicate (Theorems 2, 3) how these relations can be used to unify and extend a large class of matrix inequalities that includes as special cases the classical Hadamard, Schur, Fan and Fischer results.

In what follows $V$ will denote a fixed $n$-dimensional unitary space with inner product $(x, y)$. The $m$ th tensor space over $V$, denoted by $\otimes_{i=1}^{m} V$, is a unitary space with inner product $\left(x_{1} \otimes \cdots \otimes x_{m}, y_{1} \otimes \cdots \otimes y_{m}\right)=\prod_{i=1}^{m}\left(x_{i}, y_{i}\right)$. If $S_{m}$ is the symmetric group of degree $m$ and $\sigma \in S_{m}$ then $P(\sigma)$ will designate the permutation operator defined on $\bigotimes_{i=1}^{m} V$ by

$$
P(\sigma) x_{1} \otimes \cdots \otimes x_{m}=x_{\phi(1)} \otimes \cdots \otimes x_{\phi(m)}, \quad \phi=\sigma^{-1} .
$$

If $H$ is a subgroup of $S_{m}$ of order $h$ and $\lambda$ is a character on $H$ of degree 1 then

$$
T_{\lambda}=\frac{1}{h} \sum_{\sigma \in H} \lambda(\sigma) P(\sigma)
$$

is a hermitian idempotent operator on $\bigotimes_{i=1}^{m} V$. Its range is called a symmetry class of tensors and will be denoted by $V_{\lambda}^{m}$ in what follows.

If $A$ is a linear transformation on $V$ then $\Pi^{m}(A)$ designates the $m$ th Kronecker power of $A$. The restricted transformation $\Pi^{m}(A) \mid V_{\lambda}^{m}: V_{\lambda}^{m} \rightarrow V_{\lambda}^{m}$ is denoted by $K^{\lambda}(A)$. The map $K^{\lambda}(A)$ is called an induced mapping, e.g., if $H=S_{m}, \lambda(\sigma)=\epsilon(\sigma)$ then $V_{\lambda}^{m}=\Lambda^{m} V$ and

\footnotetext{
1 This research was supported by the U. S. Air Force under Grant AFOSR-698-65.
} 
$K^{\lambda}(A)$ is the $m$ th Grassman compound of $A$, usually denoted by $C_{m}(A)$.

Let $\Gamma_{m, n}$ denote the totality of sequences $\omega=\left(\omega_{1}, \cdots, \omega_{m}\right), 1 \leqq \omega_{i}$ $\leqq n$. Define an equivalence relation in $\Gamma_{m, n}: \omega \sim \beta$ if there exists a $\sigma \in H$ for which $\omega^{\sigma}=\left(\omega_{\sigma(1)}, \cdots, \omega_{\sigma(m)}\right)=\beta$. Let $\Delta$ be a system of distinct representatives in $\Gamma_{m, n}$ chosen so that each element $\omega \in \Delta$ is first in its equivalence class in lexicographic order. Let $\bar{\Delta}$ be the set of elements $\omega \in \Delta$ for which

$$
\sum_{\sigma \in H, \omega^{\sigma}=\omega} \lambda(\sigma)=\nu(\omega) \neq 0 .
$$

In other words (since $\lambda$ is a character of degree 1 ), a sequence $\omega \in \Delta$ is in $\bar{\Delta}$ if and only if the character $\lambda$ is identically 1 on the subgroup of $H$ consisting of all those $\sigma$ for which $\omega^{\sigma}=\omega$, e.g., if $m \leqq n, H=S_{m}$, $\lambda(\sigma)=\epsilon(\sigma)$, then $\bar{\Delta}$ is the set of all increasing sequences $\omega, 1 \leqq \omega_{1}$ $<\cdots<\omega_{m} \leqq n$, and $\nu(\omega)=1$. This set is usually denoted by $Q_{m, n}$. Again, if $m \leqq n, H=S_{m}, \lambda=1$ then $\Delta=\bar{\Delta}=G_{m, n}$, the totality of nondecreasing sequences $\omega, 1 \leqq \omega_{1} \leqq \cdots \leqq \omega_{m} \leqq n$. In this case $\nu(\omega)$ is the product of the factorials of the multiplicities of the distinct integers appearing in $\omega$, e.g., $\nu(2,2,2,3,3,8,8,8)=3 ! 2 ! 3 !$. In general $m_{i}(\omega)$ will denote the multiplicity of occurrence of $i$ in $\omega$.

If $x_{i} \in V, i=1, \cdots, m$, then $x_{1} * \cdots * x_{m}$ will denote the element $T_{\lambda}\left(x_{1} \otimes \cdots \otimes x_{m}\right)$ in $V_{\lambda}^{m}$. It is easy to check that $K^{\lambda}(A) x_{1} * \cdots$ $* x_{m}=A x_{1} * \cdots * A x_{m}$. If $x_{1}, \cdots, x_{n}$ are in $V$ and $\gamma \in \Gamma_{m, n}$ then $x_{\gamma}^{*}$ will denote $x_{\gamma_{1}} * \cdots * x_{\gamma_{m}}$. One can verify directly that if $v_{1}, \cdots, v_{n}$ is an orthonormal basis in $V$ then the tensors $(h / \nu(\gamma))^{1 / 2} v_{\gamma}^{*}$, $\gamma \in \bar{\Delta}$, form an orthonormal basis of $V_{\lambda}^{m}$.

2. Results. The main result relating two orthonormal bases in $V_{\lambda}^{m}$ can be stated as follows. We assume here that $m \leqq n$.

THEOREM 1. Let $v_{1}, \cdots, v_{n}$ and $e_{1}, \cdots, e_{n}$ be two orthonormal bases in $V$. Then for each $\omega \in \bar{\Delta}$ and each $t, 1 \leqq t \leqq n$,

(1) $\sum_{i=1}^{n} m_{i}(\omega)\left|\left(e_{i}, v_{t}\right)\right|^{2}=\sum_{\gamma \in \bar{\Delta}} m_{t}(\gamma)\left|\left(\left(\frac{h}{\nu(\omega)}\right)^{1 / 2} e_{\omega}^{*},\left(\frac{h}{\nu(\gamma)}\right)^{1 / 2} v_{\gamma}^{*}\right)\right|^{2}$.

The special case of this result when $H=S_{m}$ and $\lambda \equiv 1$ will appear in a forthcoming paper [5].

Let

$$
c_{\omega, \gamma}=\left|\left(\left(\frac{h}{\nu(\omega)}\right)^{1 / 2} e_{\omega}^{*},\left(\frac{h}{\nu(\gamma)}\right)^{1 / 2} v_{\gamma}^{*}\right)\right|^{2}, \quad \omega, \gamma \in \Xi,
$$


and let $N=\operatorname{dim} V_{\lambda}^{m}$. Then of course $\left(c_{\omega, \gamma}\right)$ is orthostochastic and hence satisfies

$$
\sum_{\gamma \in \bar{\Delta}} c_{\omega, \gamma}=1, \quad \sum_{\omega \in \bar{\Delta}} c_{\omega, \gamma}=1
$$

for each $\omega$ and $\gamma$ in $\bar{\Delta}$ respectively. However, the identity (1) tells us that in addition to (2) the $c_{\omega, \gamma}$ satisfy

$$
\sum_{i=1}^{n} m_{i}(\omega)\left|\left(e_{i}, v_{t}\right)\right|^{2}=\sum_{\gamma \in \bar{\Delta}} m_{t}(\gamma) c_{\omega, \gamma}
$$

If $X$ is an $m$-square matrix then the matrix function $d_{\lambda}$ associated with the symmetry operator $T_{\lambda}$ is defined by

$$
d_{\lambda}(X)=\sum_{\sigma \in H} \lambda(\sigma) \prod_{i=1}^{m} x_{i \sigma(i)} .
$$

The relation between $d_{\lambda}$ and $T_{\lambda}$ is as follows:

$$
\left(x_{1} * \cdots * x_{m}, y_{1} * \cdots * y_{m}\right)=\frac{1}{h} d_{\lambda}\left(\left(x_{i}, y_{j}\right)\right) .
$$

As mentioned in the introduction, Theorem 1 can be used to unify several classical results.

THEOREM 2. Let $A$ be a positive definite hermitian transformation on $V$ with eigenvalues $\alpha_{1} \geqq \alpha_{2} \geqq \cdots \geqq \alpha_{n}$. Let $\omega \in \bar{\Delta}$ and let $p_{1} \geqq p_{2}$ $\geqq \cdots \geqq p_{r} \geqq 1$ be the multiplicities of the distinct integers appearing in $\omega$. Then if $e_{1}, \cdots, e_{n}$ is any orthonormal basis of $V$

$$
d_{\lambda}\left(\left(A e_{\omega_{i}}, e_{\omega_{j}}\right)\right) \geqq \nu(\omega) \prod_{i=1}^{r} \alpha_{n-i+1}^{p_{i}}
$$

We describe a number of specializations of Theorem 2. To begin with take $H$ to be the identity subgroup of $S_{m}$. Then $d_{\lambda}(X)$ collapses to $\prod_{i=1}^{m} x_{i i}$. Take $\omega \in Q_{m, n}$ so that $p_{1}=\cdots=p_{m}=1$ and set $x_{i}=e_{\omega_{i}}$ to conclude from (5) that

$$
\prod_{i=1}^{m}\left(A x_{i}, x_{i}\right) \geqq \prod_{i=1}^{m} \alpha_{n-i+1}
$$

for any orthonormal vectors $x_{1}, \cdots, x_{m}$. This result is due to Fan [2].

Next, let $H$ be arbitrary and let $m=n$. Then if $\omega=(1, \cdots, n)$, $\nu(\omega)=1$ and (5) becomes 


$$
d_{\lambda}(B) \geqq \operatorname{det}(B),
$$

where $B=\left(\left(A e_{i}, e_{j}\right)\right)$. This is Schur's result [6] on the generalized matrix function $d_{\lambda}$.

By letting $H=S_{m}$ and $\lambda \equiv 1$ we can also specialize (5) to obtain for the permanent function,

$$
\operatorname{per}(K[\omega \mid \omega]) \geqq p_{1} ! \cdots p_{r} ! \prod_{i=1}^{r} \alpha_{n-i+1}^{p_{i}}
$$

Here $K$ is an $n$-square positive definite hermitian matrix with eigenvalues $\alpha_{1} \geqq \cdots \geqq \alpha_{n}$ and $K[\omega \mid \omega]$ is the $m$-square matrix whose $(i, j)$ entry is $k_{\omega_{i}, \omega_{j}}, i, j=1, \cdots, m$. We remark that (8) cannot be obtained from Schur's inequality ( 7 ) because the appearance of multiplicities in $\omega$ implies that $\operatorname{det}(K[\omega \mid \omega])=0$.

The next result contains the Fischer inequality as a special case.

Theorem 3. Let $A$ be an n-square positive definite hermitian matrix. For each $i, 1 \leqq i \leqq s$, let $\omega^{i}=\left(\omega_{1}^{i}, \cdots, \omega_{n_{i}}^{i}\right)$ be a strictly increasing sequence of integers in $1, \cdots, n$, having the following property: each integer $j, 1 \leqq j \leqq n$, appears $k$ times altogether among $\omega^{1}, \cdots, \omega^{s}$ and $k$ is the same for all $j$. Then

$$
\prod_{i=1}^{\dot{x}} \operatorname{det}\left(A\left[\omega^{i} \mid \omega^{i}\right]\right) \geqq(\operatorname{det}(A))^{k}
$$

In case $s=2$ and $\omega^{1}$ and $\omega^{2}$ are nonoverlapping sequences it follows that $k=1$ and (9) becomes the Fischer inequality.

3. Proof outlines. The idea behind the proof of Theorem 1 is to construct a normal transformation $N$ on $V$ with fixed orthonormal eigenvectors $v_{1}, \cdots, v_{n}$ and variable eigenvalues $\alpha_{1}, \cdots, \alpha_{n}$. Using the spectral theorem for $K^{\lambda}(N)$ we can write

$$
\left(K^{\lambda}(N) e_{\omega}^{*}, e_{\omega}^{*}\right)=\sum_{\gamma \in \bar{\Delta}} \prod_{t=1}^{n} \alpha_{t}^{m_{t}(\gamma)}\left|\left(e_{\omega}^{*},\left(\frac{h}{\nu(\gamma)}\right)^{1 / 2} v_{\gamma}^{*}\right)\right|^{2}
$$

The rest of the argument is tedious and consists of equating the partial derivatives of both sides of (10) with respect to $\alpha_{t}$, $t=1, \cdots, n$, evaluated for $\alpha_{1}=\cdots=\alpha_{n}=1$.

We proceed to a more detailed proof of Theorem 2 .

Let $v_{1}, \cdots, v_{n}$ be an o.n. set of eigenvectors of $A$ corresponding respectively to $\alpha_{1}, \cdots, \alpha_{n}$. Then from (4), 


$$
\begin{aligned}
d_{\lambda}\left(\left(A e_{\omega_{i}}, e_{\omega_{j}}\right)\right) & =h\left(K^{\lambda}(A) e_{\omega}^{*}, e_{\omega}^{*}\right) \\
& =\nu(\omega) \sum_{\gamma \in \bar{\Delta}} \prod_{t=1}^{n} \alpha_{t}^{m_{t}(\gamma)}\left|\left(\left(\frac{h}{\nu(\omega)}\right)^{1 / 2} e_{\omega}^{*},\left(\frac{h}{\nu(\gamma)}\right)^{1 / 2} v_{\gamma}^{*}\right)\right|^{2} \\
& =\nu(\omega) \sum_{\gamma \in \bar{\Delta}} \prod_{t=1}^{n} \alpha_{t}^{m_{t}(\gamma)} c_{\omega, \gamma} \\
& \geqq \nu(\omega) \prod_{\gamma \in \bar{\Delta}} \prod_{t=1}^{n} \alpha_{t}^{m_{t}(\gamma) c_{\omega, \gamma}} \\
& =\nu(\omega) \prod_{t=1}^{n} \alpha_{t}^{\Sigma_{\gamma \in \bar{\Delta}^{m}}(\gamma) c_{\omega, \gamma}} \\
& =\nu(\omega) \prod_{t=1}^{n} \alpha_{t}^{\Sigma_{i=1}^{n} m_{i}(\omega)\left|\left(e_{i}, v t\right)\right|^{2}} .
\end{aligned}
$$

The inequality in (11) is just the weighted arithmetic-geometric mean inequality and the last equality in (11) is the identity (3). The $n$-square matrix $\left(\left|\left(e_{i}, v_{t}\right)\right|^{2}\right)$ is orthostochastic and a fairly routine argument using the Birkhoff theorem [4, p. 112] shows that

$$
\prod_{t=1}^{n} \alpha_{t}^{\Sigma_{i-1}^{n} m_{i}(\omega)\left|\left(e_{i}, v_{t}\right)\right|^{2}} \geqq \prod_{i=1}^{r} \alpha_{n-i+1}^{p_{i}} \text {. }
$$

The proof of Theorem 3 consists of the same kinds of calculations.

\section{REFERENCES}

1. C. Chevalley, The construction and study of certain important algebras, The Mathematical Society of Japan, Tokyo, 1955.

2. K. Fan, Problem 4429, Amer. Math. Monthly 58 (1951), 194.

3. W. V. D. Hodge and D. Pedoe, Methods of algebraic geometry, Vol. 1, Cambridge, Univ. Press, New York, 1947.

4. Marvin Marcus and Henryk Minc, $A$ survey of matrix theory and matrix inequalities, Allyn and Bacon, Boston, 1964.

5. — Generalized matrix functions, Trans. Amer. Math. Soc. (to appear).

6. I. Schur, Über endliche Gruppen und Hermitesche Formen, Math. Z. 1 (1918), 184-207.

University of California, Santa Barbara 